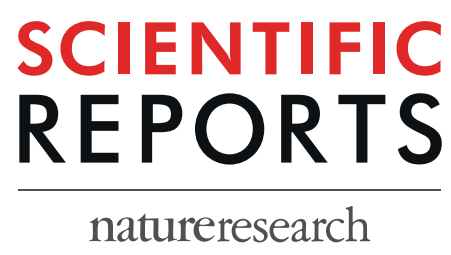

\title{
OPEN Mucoadhesive effect of Curcuma longa extract and curcumin decreases the ranitidine effect, but not bismuth subsalicylate on ethanol-induced ulcer model
}

\author{
Alejandra Orona-Ortiz $\mathbb{1}^{1}$, Luis Medina-Torres $\mathbb{C}^{1}{ }^{1}$, Josué A. Velázquez-Moyado $\mathbb{C}^{1}$, \\ Elizabeth A. Pineda-Peña $\mathbb{D}^{1}$, José Luis Balderas-López $\mathbb{1}^{1}{ }^{1}$, María Josefa Bernad-Bernad ${ }^{1}$, \\ José Carlos Tavares Carvalho $\mathbb{1}^{2}$ \& Andrés Navarrete $\mathbb{1}^{1 *}$
}

The study of pharmacological interactions between herbal remedies and conventional drugs is important because consuming traditional herbal remedies as supplements or alternative medicine is fairly common and their concomitant administration with prescribed drugs could either have a favorable or unfavorable effect. Therefore, this work aims to determine the pharmacological interactions of a turmeric acetone extract (TAE) and its main metabolite (curcumin) with common anti-ulcer drugs (ranitidine and bismuth subsalicylate), using an ethanol-induced ulcer model in Wistar rats. The analysis of the interactions was carried out via the Combination Index-Isobologram Equation method. The combination index $(\mathrm{Cl})$ calculated at 0.5 of the affected fraction $(f a)$ indicated that the TAE or curcumin in combination with ranitidine had a subadditive interaction. The results suggest that this antagonistic mechanism is associated to the mucoadhesion of curcumin and the TAE, determined by rheological measurements. Contrastingly, both the TAE and curcumin combined with bismuth subsalicylate had an additive relationship, which means that there is no pharmacological interaction. This agrees with the normalized isobolograms obtained for each combination. The results of this study suggest that mucoadhesion of curcumin and the TAE could interfere in the effectiveness of ranitidine, and even other drugs.

For centuries, medicinal plants were the primary source of health care. Herbal remedies are still used by $\sim 70 \%$ of the population, according to the World Health Organization $(\mathrm{WHO})^{1}$. Some studies have reported that despite the lack of information about the efficacy and safety regarding alternative and traditional herbal medicine, their use is increasing. Therefore, it is essential to investigate possible interactions between herbal treatments and conventional drugs. When simultaneously consuming herbal and drug treatments, there may be negative interactions that diminish the effectiveness of the treatment, or could even present adverse symptomatology ${ }^{1-3}$. However, some interactions could be favorable to the patient, enhancing the therapeutic activity ${ }^{4}$.

Turmeric root (Curcuma longa L. [Zingiberaceae]) is used in Ayurveda and Chinese traditional medicine as a remedy for peptic ulcer ${ }^{5}$. Several studies show the gastroprotective activity of Curcuma extracts in different ulcer models in rats. These extracts also accelerate the gastric mucosal healing, decrease the acid release and increase the expression of cyclooxygenase-2 (COX-2) and superoxide dismutase (SOD) ${ }^{6-8}$. Curcumin (Fig. 1), one of the major components of $C$. long $a^{9,10}$, improves ulceration healing by the restitution of collagen fibers and angiogenesis stimulation ${ }^{11}$. It has been reported that the ethyl acetate extract of Curcuma longa prevents the gastric mucosal damage in pylori-ligated rats, decreasing the acid release by antagonizing histamine- 2 receptors; however,

${ }^{1}$ Facultad de Química, Departamento de Farmacia, Universidad Nacional Autónoma de México. Ciudad Universitaria Coyoacán, 04510, Ciudad de Mexico, Mexico. 'Laboratorio de Pesquisa em Farmacos, Curso de Farmacia, Departamento de Ciências Biólogicas e da Saúde, Universidade Federal do Amapá, Macapá, AP, Brazil. *email: anavarrt@unam.mx 
<smiles>COc1cc(/C=C/C(=O)/C=C(\O)C=Cc2cccc(O)c2)ccc1O</smiles>

Figure 1. Chemical structure of curcumin.

\begin{tabular}{|c|c|c|c|c|c|c|c|c|c|}
\hline & Dm Exp (mg/kg) & Dm Theo (mg/kg) & $\mathbf{m}$ & \multicolumn{2}{|l|}{$\mathrm{CI}_{10}$} & \multicolumn{2}{|l|}{$\mathrm{CI}_{50}$} & \multicolumn{2}{|l|}{$\mathrm{CI}_{90}$} \\
\hline TAE & 0.004 & & 0.21 & & & & & & \\
\hline Curcumin & 0.99 & & 0.38 & & & & & & \\
\hline Ranitidine & 17.40 & & 0.62 & & & & & & \\
\hline Bismuth subsalicylate & 11.50 & & 1.49 & & & & & & \\
\hline TAE-Ranitidine & 31.11 & 8.70 & 1.19 & 7196 & Ant & 3.57 & Ant & 0.32 & Syn \\
\hline Curcumin-Ranitidine & 31.79 & 9.19 & 0.74 & 27.65 & Ant & 3.37 & Ant & 1.12 & Add \\
\hline TAE-Bismuth subsalicylate & 6.35 & 5.75 & 0.98 & 1499 & Ant & 1.10 & Add & 1.17 & Add \\
\hline Curcumin-Bismuth subsalicylate & 8.02 & 6.24 & 1.16 & 31.35 & Ant & 1.28 & Add & 0.99 & Add \\
\hline
\end{tabular}

Table 1. Median dose $(\mathrm{Dm})$ and graph shape $(\mathrm{m})$ obtained from the median-effect plot, for each drug and combination. Combination index $(C I)$ values for low $\left(C I_{10}\right)$, medium $\left(C I_{50}\right)$ and high $\left(C I_{90}\right)$ level of $f a$ effect. $D m$ Exp and $D m$ Theo are the experimental and theoretical $D m$ values in the combinations. Add indicates additive effect $(C I=1)$, Ant indicates antagonism $(C I>1)$ and Syn indicates synergism $(C I<1) . C I_{10}, C I_{50}$, and $C I_{90}$ are the $C I$ calculated for the combinations at $0.1,0.5$ and 0.9 affected fractions $(f a)$.

curcumin does not show this antagonistic effect ${ }^{6}$. Nevertheless, the antioxidant effect of curcumin could help to prevent ethanol-induced gastric damage due to its scavenging activity on reactive oxygen species (ROS) $)^{12,13}$.

Peptic ulceration is defined as a gastric mucous membrane injury in the stomach or duodenum, with the sloughing of inflamed dead tissue ${ }^{14}$. In Mexico, around 1'500,000 cases of gastric ulcer, gastritis and duodenitis are annually reported ${ }^{15}$. Despite that Proton Pump Inhibitors (PPIs) are the most common worldwide treatment for peptic ulcer, it is reported that omeprazole increases duodenal ethanol injury in rats ${ }^{16}$. Under our experimental conditions, using an ethanol-induced gastric damage model, omeprazole, did not show a dose-dependent effect, so construction of the dose-response curve was not possible. Therefore, in this study, we decided to evaluate another over-the-counter (OTC) drugs used in ulcer treatment. Ranitidine is a histamine-2 receptor antagonist, which decreases acid secretion ${ }^{17}$, and bismuth subsalicylate protects the gastric mucosa from damage by covering and protecting the stomach from luminal acid and increases the mucus secretion ${ }^{18}$; it has also been reported that it protects against stress, an inadequate diet and alcohol-induced injuries ${ }^{19}$.

Additionally, the turmeric market has increased around the world ${ }^{5}$; therefore, the concomitant use of curcuma with conventional antiulcer drugs is likely among patients with gastric ailments. It is of great importance to study pharmacological interactions because it is not usually known how the organism will respond to a multi-drug treatment. The effect of a given combination may be simply the addition of the individual effects (additivity), or there could be an increase or attenuation of the activity. The enhanced effect is called superadditive or synergistic (favorable interactions), and the attenuated one subadditive (unfavorable interactions) ${ }^{20}$. Favorable interactions may help to diminish the drug doses and their side effects. When finding an unfavorable interaction, drug combinations should be adequately handled.

Continuing with our systematic study on the pharmacological interactions between natural products and conventional drugs ${ }^{21-23}$; in this study, we investigated the acetone extract of C. longa (TAE) and curcumin, its major active metabolite, in combination with ranitidine and bismuth subsalicylate, using an ethanol-induced gastric injury model in Wistar rats ${ }^{24}$. We used the Combination Index-Isobologram Equation analysis described by Chou and Talalay in 1976 to assess the effects of the combined treatments ${ }^{20}$.

\section{Results}

The curcumin and TAE were more potent inhibitors of the gastric damage (Table 1), with lower Dm values (TAE $0.004 \mathrm{mg} / \mathrm{kg}$; curcumin $0.99 \mathrm{mg} / \mathrm{kg}$ ) than those of ranitidine and bismuth subsalicylate $(17.4 \mathrm{and} 11.5 \mathrm{mg} / \mathrm{kg})$. The calculated $D m$ were used to establish the dose-proportion in each combination, according to the experimental design (Table 2). The median-effect plots for each combination (Fig. 2) were used to calculate the $\mathrm{Dm}$ and $m$ using Eq. 4 for both combinations (Table 1). Figure 3 shows representative pictures of stomachs for each treatment, in which the macroscopic gastric damage caused by absolute ethanol is observed. All treatments showed less damaged area $\left(\mathrm{mm}^{2}\right)$ than the ulcerated control group also, it is important to notice that the combinations TAE-ranitidine and curcumin-ranitidine showed more damaged area than the other treatments.

When TAE and curcumin were administrated before ranitidine, the combinations showed a subadditive interaction (Figs 3 and 4A,B). However, when the administration sequence was inverted, there was an additive effect 


\begin{tabular}{|l|l|l|l|l|l|l|l|}
\hline & & \multicolumn{7}{|c|}{ Drug 1 } \\
\hline & & 0 & $0.25 \times(D m)_{1}$ & $0.5 \times(D m)_{1}$ & $(D m)_{1}$ & $2 \times(D m)_{1}$ & $4 \times(D m)_{1}$ \\
\hline & 0 & Control & $(f a)_{1}$ & $(f a)_{1}$ & $(f a)_{1}$ & $(f a)_{1}$ & $(f a)_{1}$ \\
\hline & & $(f a)_{0}$ & & & & & \\
\hline \multirow{5}{*}{ Drug 2 } & $0.25 \times(D m)_{2}$ & $(f a)_{2}$ & $(f a)_{1,2}$ & & & & \\
\cline { 2 - 9 } & $0.5 \times(D m)_{2}$ & $(f a)_{2}$ & & $(f a)_{1,2}$ & & & \\
\cline { 2 - 9 } & $(D m)_{2}$ & $(f a)_{2}$ & & & $(f a)_{1,2}$ & & \\
\cline { 2 - 9 } & $2 \times(D m)_{2}$ & $(f a)_{2}$ & & & & $(f a)_{1,2}$ & \\
\cline { 2 - 9 } & $4 \times(D m)_{2}$ & $(f a)_{2}$ & & & & & $(f a)_{1,2}$ \\
\hline
\end{tabular}

Table 2. Experimental design for the evaluation of 1:1 combinations. $D m=$ median dose; $f a=$ affected fraction. Drug $1=$ curcumin or TAE. Drug $2=$ ranitidine or bismuth subsalicylate.

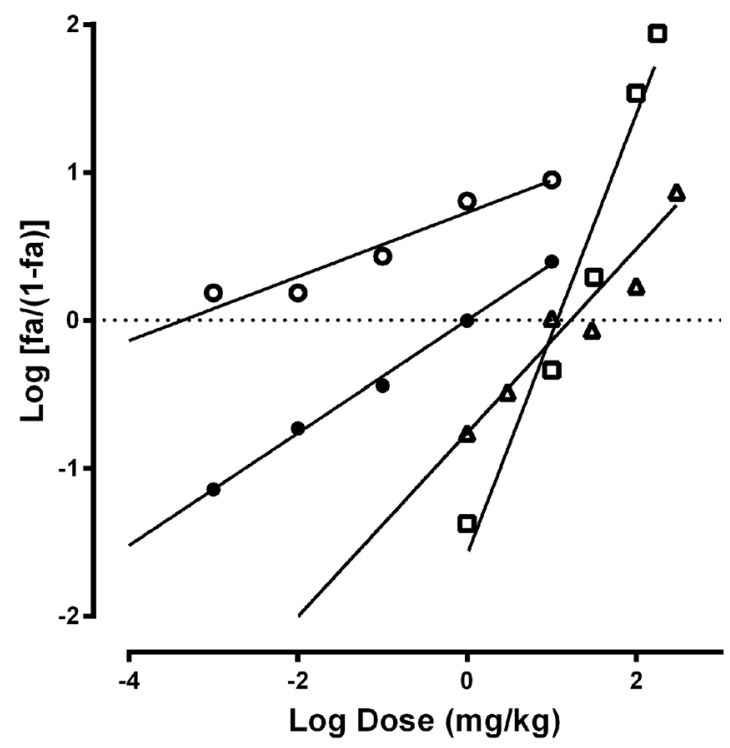

Figure 2. Median effect-plot of the single treatments: TAE (o) $D m: 0.004 \mathrm{mg} / \mathrm{kg}$, curcumin (๑) Dm: 0.99 mg/kg, ranitidine $(\triangle) D m: 17.40 \mathrm{mg} / \mathrm{kg}$ and bismuth subsalicylate $(\square) D m: 11.50 \mathrm{mg} / \mathrm{kg}$.

(Fig. 4C,D). Figure 5 shows the combination index $(C I)$ calculated for the combinations of TAE and curcumin with ranitidine. The $C I$ for $0.5 \mathrm{fa}$ indicates there is a subadditive interaction; which agrees with the isobolographic analysis (Fig. 4).

The observed subadditive effect may be attributed to the fact that TAE and curcumin coat the mucosal layer of the stomach, forming a protective barrier. This barrier could interfere with ranitidine absorption, thus reducing its gastroprotective effect. To further analyze the antagonist behavior of the TAE and curcumin against the ranitidine effect, we decided to indirectly evaluate the mucoadhesion. Figure 6 shows that mixtures of TAE-mucin and curcumin-mucin have rheological synergy (positive $\Delta \mathrm{G}^{\prime}$ and $\Delta \mathrm{G}^{\prime}$ ). This indicates a strong interaction between the components of the mixture, which is related to mucoadhesion. The oscillatory flux-plots (Fig. 6A,B) show a "pseudo-solid" behavior under the testing conditions at all frequencies $\left(\Delta \mathrm{G}^{\prime \prime}>\Delta \mathrm{G}^{\prime}\right)$, suggesting a strong interaction between the continuous and dispersed phases. The higher synergy values were observed at high frequencies (Fig. 6C,D).

The combination of curcumin or TAE with bismuth subsalicylate does not show any pharmacological interaction. The CI-plots of these combined treatments (Fig. 7) correspond to an additive interaction for $f a \geq 0.5$ and subadditive for $f a<0.5$. The normalized isobolograms of the bismuth subsalicylate with TAE and curcumin combined treatments showed an additive effect in both cases (Fig. 8). The experimental Dm falls within the additive area of the isobolograms and there is no statistical difference between the experimental ( $D m$ Exp) and theoretical Dm (Dm Theo) (Table 1).

\section{Discussion}

The gastroprotective effect of turmeric and ranitidine proceeds via different mechanisms of action. For the Curcuma species, the molecular mechanism involves antioxidant and anti-inflammatory activities, regulating the most important inflammatory modulators: the inducible nitric oxide synthase (iNOS), tumor necrosis factor-alpha $(\mathrm{TNF}-\alpha)$ and nuclear factor kappa-B $(\mathrm{NF}-\kappa \mathrm{B})^{10}$. Ranitidine is an antagonist of the histamine- 2 receptors that reduces the secretion of hydrochloric acid and pepsin ${ }^{17}$. Interestingly, Kim et al. (2005) reported that the Curcuma longa ethyl acetate extract decreases the acid release because of its antagonistic effect on histamine-2 receptors in pylori-ligated rats, but curcumin did not show this antagonistic effect ${ }^{6}$. Therefore, it was necessary to 

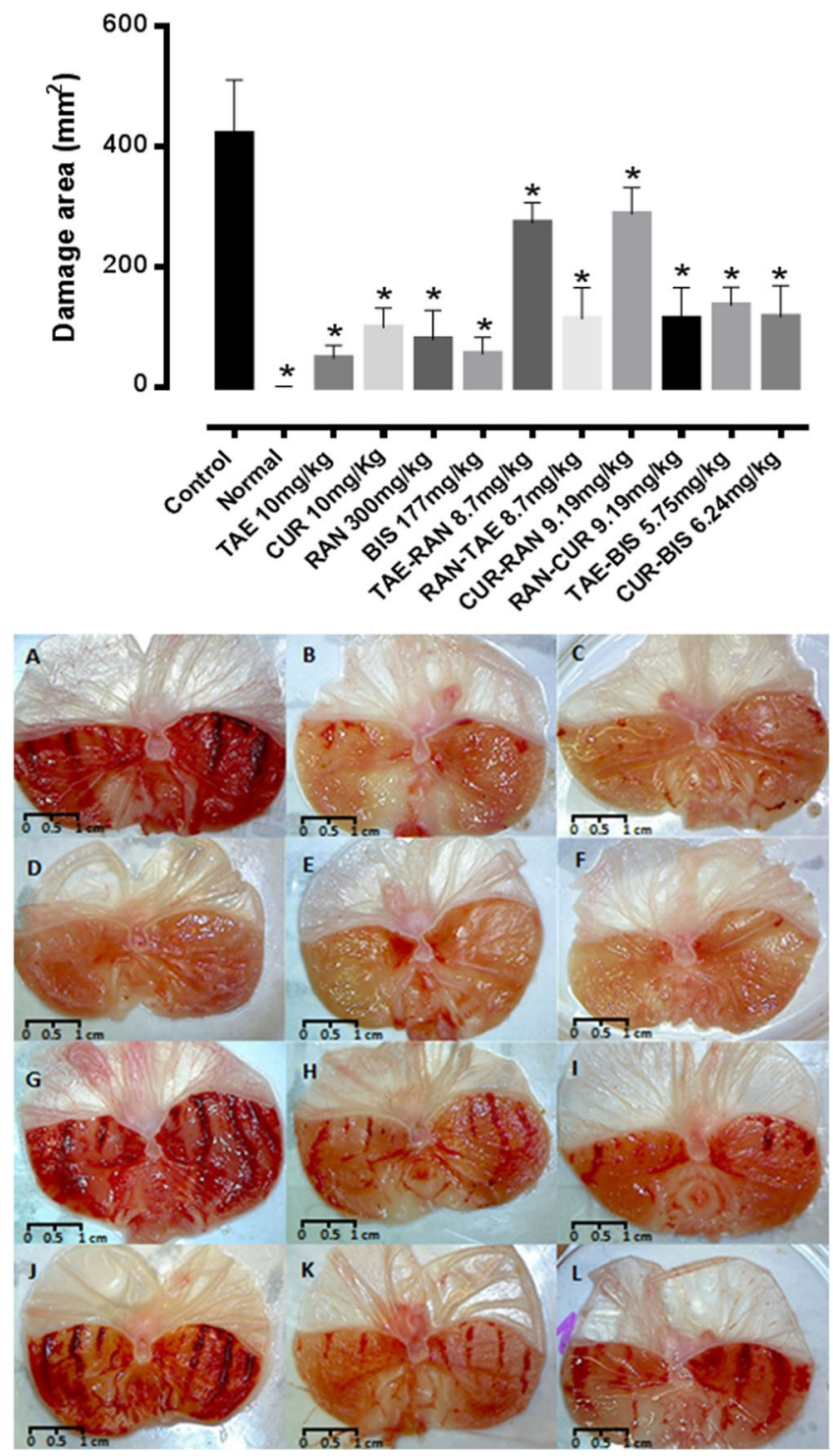

Figure 3. Damage areas $\left(\mathrm{mm}^{2}\right)$ of the representative treatments. *The statistically significant difference $(p<0.05)$ between the treatments and the control was determined by a One-way ANOVA and a post hoc Dunnett test. Representative stomachs for each treatment: (A) control (absolute ethanol); (B) TAE (10 mg/kg), (C) curcumin $(10 \mathrm{mg} / \mathrm{kg}) ;(\mathbf{D})$ normal stomach; (E) ranitidine $(300 \mathrm{mg} / \mathrm{kg}) ;$ (F) bismuth subsalicylate $(177 \mathrm{mg} /$ $\mathrm{kg}$ ); (G) TAE-ranitidine 1:1 and (H) ranitidine-TAE 1:1 (Dmteo $8.7 \mathrm{mg} / \mathrm{kg}) ;(\mathbf{J})$ curcumin-ranitidine 1:1 and (K) ranitidine-curcumin 1:1 (Dmteo $9.19 \mathrm{mg} / \mathrm{kg})$; (I) TAE-bismuth subsalicylate 1:1 (Dmteo $5.75 \mathrm{mg} / \mathrm{kg})$ and $(\mathbf{L})$ curcumin-bismuth subsalicylate 1:1 (Dmteo $6.24 \mathrm{mg} / \mathrm{kg}$ ).

evaluate the interaction of the turmeric extract and its principal metabolite, curcumin, with ranitidine to determine whether the concomitant administration is favorable.

The interaction analysis, performed via the combination index and isobologram method ${ }^{20}$, shows that when the extract or the curcumin is administered before ranitidine (in 1:1 proportion), there is a subadditive gastroprotective effect (Fig. 5). Furthermore, the isobolographic analysis demonstrates that the Dm Exp of the combination with TAE or curcumin $(31.11$ and $31.79 \mathrm{mg} / \mathrm{kg}$, respectively) was higher than the theoretical value $(8.70$ and $9.19 \mathrm{mg} / \mathrm{kg}$ ), revealing a statistically significant difference (Fig. 4A,B).

When the rat stomachs were dissected, we observed the characteristic yellow color of both the extract and curcumin coating the mucosal layer. In order to evaluate a possible physicochemical interaction that would explain the antagonistic effect of the extract or the curcumin with ranitidine; the combinations were tested in vitro $\left(0.1 \mathrm{M} \mathrm{HCl}, 37^{\circ} \mathrm{C}, 2 \mathrm{~h}\right)$. The LC/MS analysis did not show any changes in the retention time or new signals that could suggest a chemical interaction (see supplemental information). As mentioned above, the barrier formed by TAE or curcumin could interfere with the gastroprotective effect of ranitidine. To assess this, we evaluated the mucoadhesion of the extract and the curcumin using a rheological method. The results shown in Fig. 6 suggest that both treatments present mucoadhesion in a simulated gastric medium ( $\mathrm{pH}$ 1.6). The rheological parameters show synergism (positive $\Delta \mathrm{G}^{\prime}$ and $\Delta \mathrm{G}^{\prime \prime}$ ) in the TAE-mucin and curcumin-mucin mixtures. Some 

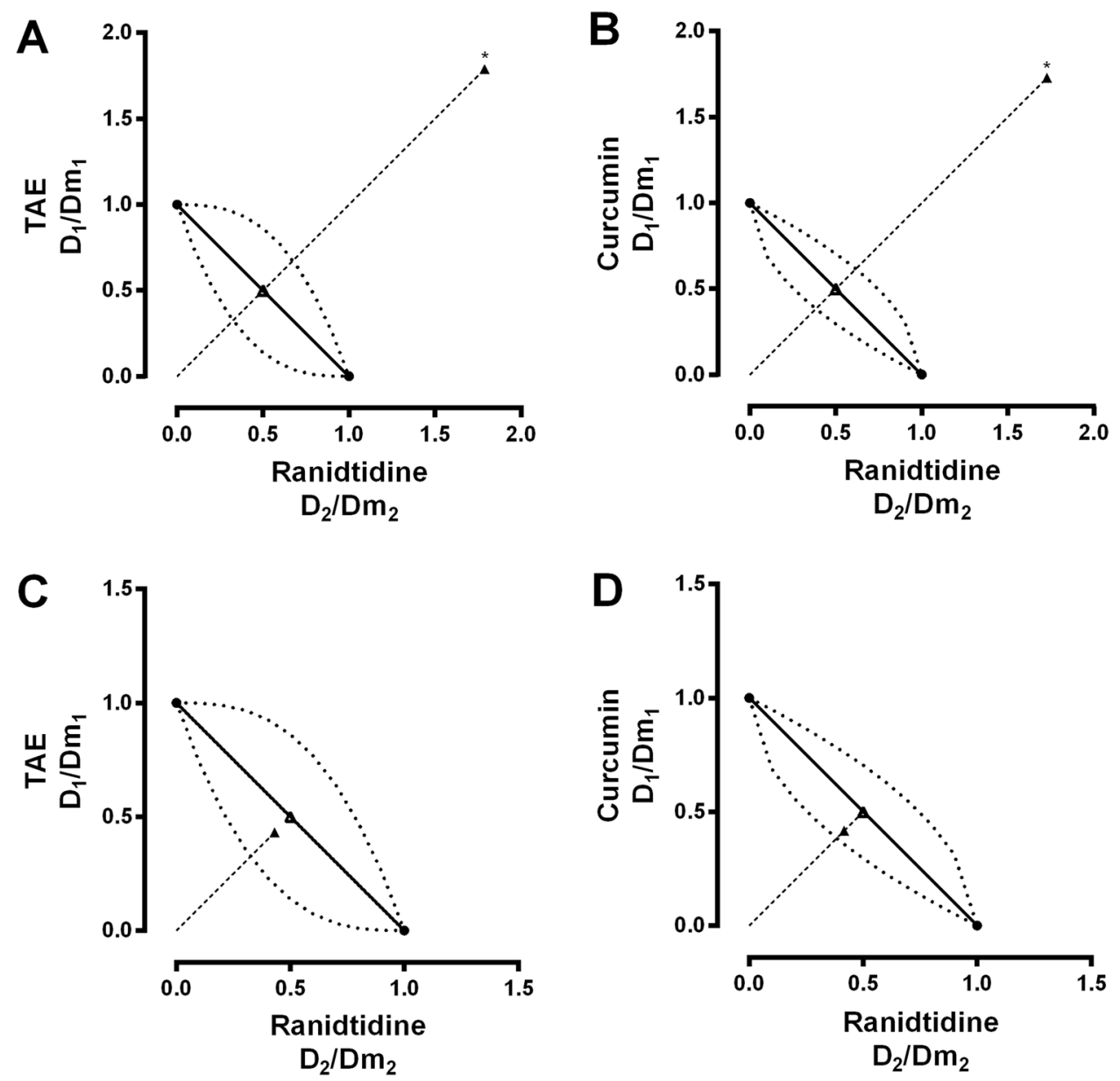

Figure 4. Normalized isobolograms of $D m$ at a 1:1 constant ratio of (A) TAE-ranitidine, (B) curcuminranitidine, (C) ranitidine-TAE (inverse administration) and (D) ranitidine-curcumin (inverse administration). The experimental $D m$ points calculated for the combinations $(\mathbf{A})$ were plotted and compared $v s$ the theoretical $D m(\triangle)$. *The statistically significant difference $(p<0.05)$ between the experimental and theoretical $D m$ was determined by the Mann-Whitney test. The concave curve and convex curve in the isobologram were calculated using Eqs 6 and 7.

A

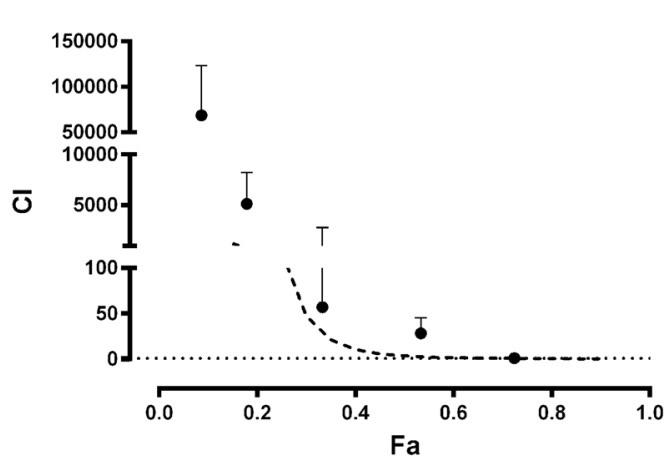

B

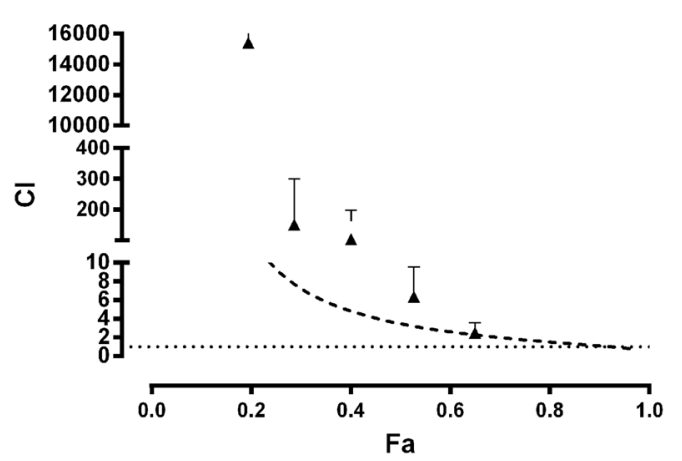

Figure 5. Calculated combination index $(C I)$ for $(\mathbf{A})$ TAE-ranitidine 1:1 (•) and (B) curcumin-ranitidine 1:1 (A). Dashed line represents the theoretical CI behavior (-). CI shows synergism, additivity or antagonism at $C I<1, C I=1$ or $C I>1$, respectively. Each point represents the mean $\pm S E M$ of at least six repetitions.

studies have reported that the "pseudo-solid" behavior of the mixtures is related to the interaction between the components of the mixture, where the viscous character dominates along the entire frequency range $\left(G^{\prime \prime}>G^{\prime}\right)$. However, in the TAE-mucin mixture, $G^{\prime \prime}$ was larger than $G^{\prime}$ with a plateau independent to frequency ${ }^{25}$, which is 

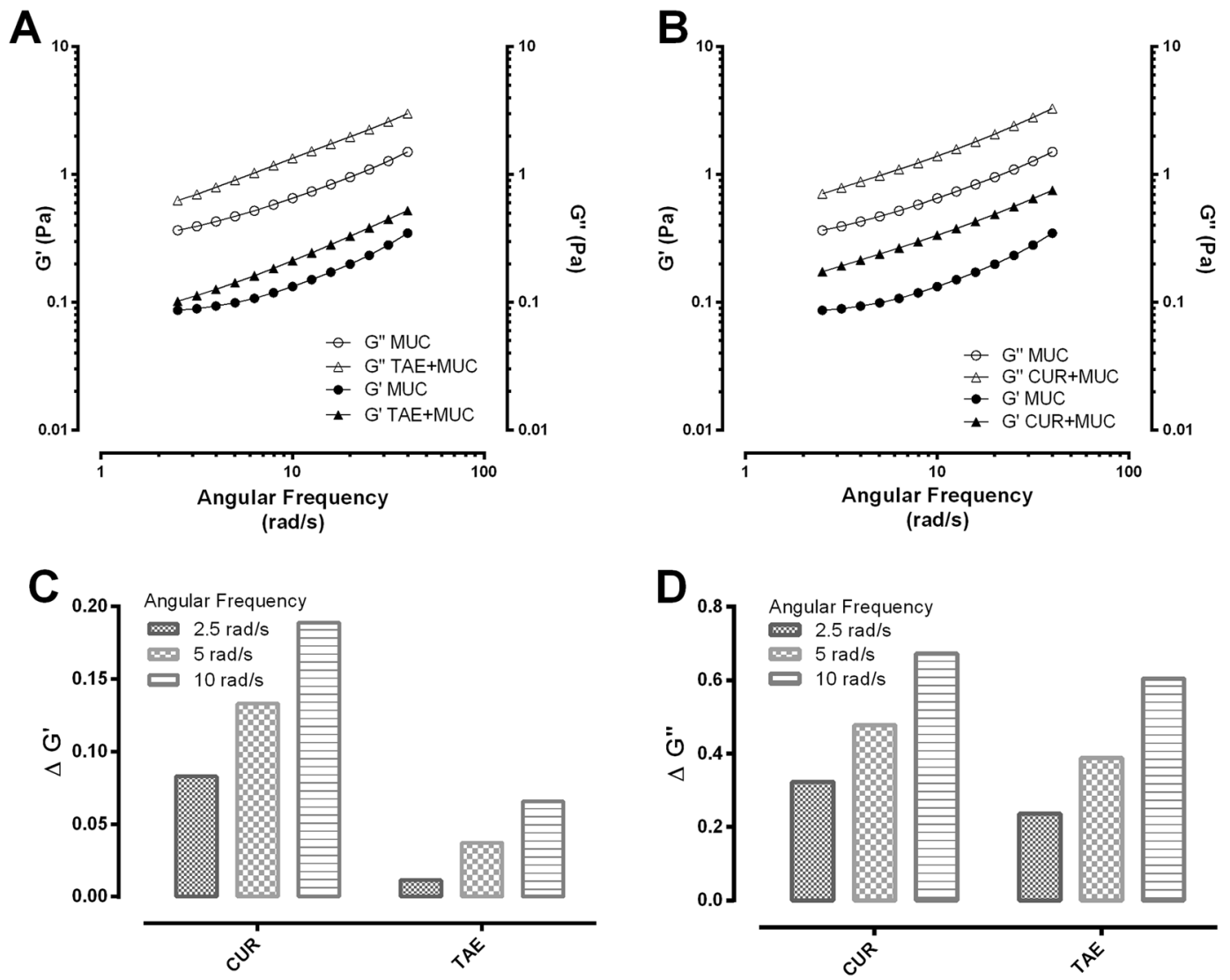

Figure 6. Frequency dependence graphics of the elastic $\left(\mathrm{G}^{\prime}\right)$ and viscous $\left(\mathrm{G}^{\prime \prime}\right)$ modulus for the mixtures of mucin with TAE (A) or curcumin (B), compared to mucin alone. (C,D) show the synergy parameters $\left(\Delta G^{\prime}\right.$ and $\left.\Delta \mathrm{G}^{\prime \prime}\right)$ at different angular frequencies $(2.5,5$ and $10 \mathrm{rad} / \mathrm{s})$ for TAE and curcumin mixtures with mucin.

A

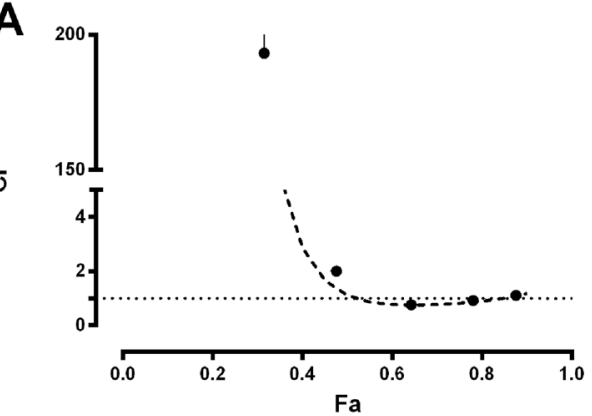

B

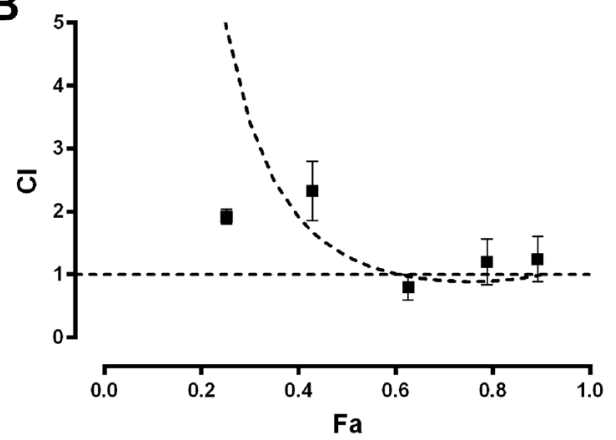

Figure 7. Calculated combination index (CI) for (A) TAE-bismuth subsalicylate 1:1 (•) and (B) curcuminbismuth subsalicylate 1:1 (ם). Dashed line represents the theoretical CI behavior (-). CI shows synergism, additivity or antagonism at $C I<1, C I=1$ or $C I>1$, respectively. Each point represents the mean \pm SEM of at least six repetitions.

possibly linked with mucoadhesion. The results of this study suggest that, in addition to the reported mechanism of action of the $C$. longa extract and curcumin, their mucoadhesion could be considered as a physical barrier mechanism related to wound and gastric ulcer healing. To corroborate whether the curcumin mucoadhesion was responsible for the antagonism, we decided to invert the sequence of administration. Ranitidine was administered $15 \mathrm{~min}$ before the curcumin to ensure its absorption. Figure $3 \mathrm{G}$,J, corresponding to the TAE-ranitidine and curcumin-ranitidine combinations, present a larger damage area than Fig. $3 \mathrm{H}, \mathrm{K}$, which are the ranitidine-TAE and ranitidine-curcumin combined treatments (inverse administration). The Dm Exp of ranitidine-TAE and ranitidine-curcumin $(7.49$ and $7.66 \mathrm{mg} / \mathrm{kg}$, respectively) was not statistically different from the $\mathrm{Dm}$ Theo $(8.70$ and $9.19 \mathrm{mg} / \mathrm{kg}$ ) (Fig. 4C); demonstrating that the physical barrier formed by the TAE or curcumin, when interacting with mucin, is responsible for the antagonism on the ranitidine effect. These results are of great relevance 

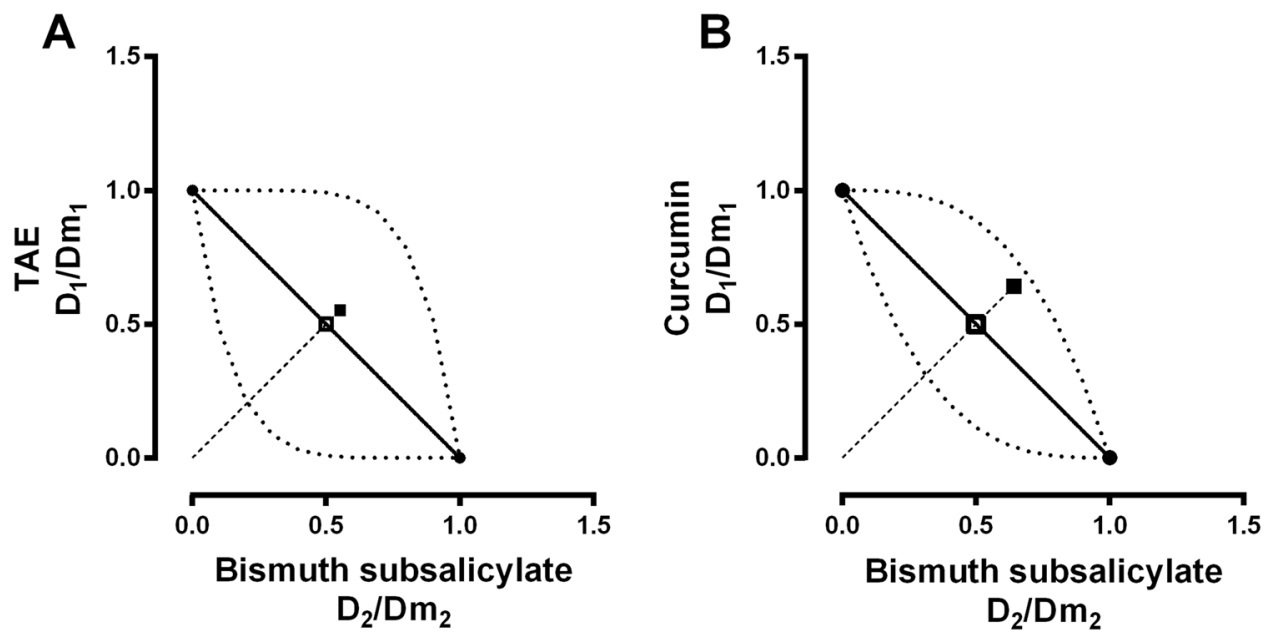

Figure 8. Normalized isobolograms of $D m$ at a 1:1 constant ratio of (A) TAE-bismuth subsalicylate and (B) curcumin-bismuth subsalicylate. The experimental $D m$ points calculated for both combinations $(\mathbf{\square})$ were plotted and compared $v s$ the theoretical $D m(\square)$. *The statistically significant difference $(p<0.05)$ between the experimental and theoretical $D m$ was determined by the Mann-Whitney test. The concave curve and convex curve in the isobologram were calculated using Eqs 6 and 7.

for patients that consume turmeric as a complementary or alternative medicine for peptic ulcer or other illnesses, as it should be recommended that the administration of the antiulcer drug occur at least 15 min before turmeric to avoid an antagonistic effect.

Contrastingly, the co-administration of TAE or curcumin with bismuth subsalicylate (1:1 fixed ratio) did not show any pharmacologic interaction (Figs 7 and 8). The Dm Exp for the combinations was not statistically different than the $\mathrm{Dm}$ Theo. The $C I$ analysis demonstrates a dual interaction effect for both combinations, antagonistic for $f a<0.5$ and additive for $f a>0.5$ (Fig. 7 and Table 1). This CI behavior indicates that doses higher than the $D m$ are adequate for the co-administration of the extract or curcumin with bismuth subsalicylate, and caution should be exerted with doses below the $\mathrm{Dm}$. In pursuance of evaluating a possible chemical interaction between the curcumin and bismuth subsalicylate, we performed an in vitro assay where both compounds were mixed for $2 \mathrm{~h}$ at their corresponding $\mathrm{Dm}$ concentration in an acid environment $\left(0.1 \mathrm{M} \mathrm{HCl} ; 37^{\circ} \mathrm{C}\right)$. Then the samples were analyzed by infrared spectroscopy. The spectra did not show any new functional groups or bonds between the compounds that would indicate a chemical interaction (see supplemental information). Therefore, it is necessary to further investigate the antagonistic mechanism observed at doses lower than the Dm. It has been suggested that prostaglandin induction and bicarbonate secretion are part of the molecular mechanism of bismuth salts; this could be involved in the CI-plot behavior ${ }^{18}$. On the other hand, the additive effect observed at the $\mathrm{Dm}$ could be related with the action mechanism of the treatments: the main mechanism of bismuth salts is coating the ulcer crater to improve wound healing, and the mucoadhesion of TAE or curcumin could also further improve the healing. In this case, both treatments share the same action mechanism; therefore, their gastroprotective behavior has an additive effect on treatments.

The ulcerative mechanism of the ethanol-induced injury model involves ROS ${ }^{26}$. The results in this work show that the extract and the curcumin are potent gastroprotective agents in this model that is likely explained by the antioxidant effect reported for curcuminoids ${ }^{10}$. The calculated $D m$ for curcumin, ranitidine and bismuth subsalicylate (Table 1) indicates that the C. longa extract is the most potent gastroprotective agent among the evaluated treatments.

\section{Conclusion}

The CI-isobologram method demonstrates that when the extract or curcumin were administered before ranitidine (1:1), there is a subadditive interaction. However, when the administration sequence was inverted, the extract or curcumin did not affect the ranitidine gastroprotective action. The rheological results suggest that the mucoadhesion of the extract and curcumin is involved in their antagonistic action mechanism. Therefore, the concomitant use of turmeric or curcumin with other drugs should be carefully evaluated; we would recommend ingesting the turmeric or curcumin at least $15 \mathrm{~min}$ after the other drugs. Our results suggest that the mucoadhesion could be the mechanism for the wound and gastric ulcer healing, in addition to those reported for turmeric and curcumin. Additionally, the combinations with bismuth subsalicylate showed an additive effect in both cases, indicating there is no pharmacologic interaction in the ethanol-induced ulceration model.

Moreover, we demonstrated that the Curcuma longa extract and curcumin are an effective treatment to prevent ethanol-induced stomach damage. Both compounds are more potent gastroprotective agents than ranitidine and bismuth subsalicylate. 


\section{Methods \\ Plant material. Dry ground powder of Curcuma longa L. root was donated by Laboratorios MIXIM, S. A. de C. V. (Brach number 11590612), from which curcumin was isolated for biological testing.}

Curcumin isolation and identification. The Curcuma longa L. acetone extract (TAE) was obtained by Soxhlet reflux of the dry and ground plant material $(1.5 \mathrm{~kg}$ ) with acetone $(3 \mathrm{~L})$ for 6 hours, yielding $270 \mathrm{~g}$ of TAE. For curcumin isolation, the extract $(100 \mathrm{~g})$ was separated by column chromatography with $1 \mathrm{~kg}$ of silica gel 60 $\left(0.063-0.200 \mathrm{~mm}\right.$, MERCK $\left.^{\circledR}\right)$. The column $(90 \times 7$ i.d. $\mathrm{cm})$ was eluted with hexane, followed by hexane: chloroform (1:1 to 1:9), then chloroform, and chloroform: methanol (99.5:0.5 to 97:3). Curcumin (Fig. 1) was isolated from the fractions eluted with chloroform and chloroform: methanol, as indicated by thin layer chromatography. The purification and crystallization of curcumin were carried out as follows. The crude curcumin was dissolved in hot methanol $\left(60^{\circ} \mathrm{C}\right)$, then ten times volume of cold hexane $\left(-20^{\circ} \mathrm{C}\right)$ was added and allowed to cool $\left(4^{\circ} \mathrm{C}\right)$ for $24 \mathrm{~h}$. The crystals were filtered and washed with cold hexane, yielding $3.9 \mathrm{~g}$ of curcumin. The melting point of the obtained compound, measured with a Fisher-Johns apparatus, agrees with the reported value ${ }^{27}$. Liquid chromatography-mass spectrometry (LC/MS) analysis indicates a 99\% purity. The mass spectrum of the curcumin was obtained by direct injection using a positive electronic impact $\left(\mathrm{EI}^{+}\right)$ionization source. The molecular ion showed a m/z $368(\mathrm{M}+1)^{+}$; the most abundant fragment ion was $\mathrm{m} / \mathrm{z} 177(\mathrm{M}+1)^{+}$, in concordance with the literature ${ }^{28}$. The ${ }^{1} \mathrm{H}$ and ${ }^{13} \mathrm{C}$ NMR spectra were recorded in deuterated methanol $\left(\mathrm{CD}_{3} \mathrm{OD}\right)$ using a Varian Unity Plus 400 spectrometer at $400 \mathrm{MHz}\left({ }^{1} \mathrm{H}\right)$ and $125 \mathrm{MHz}\left({ }^{13} \mathrm{C}\right)$, using tetramethylsilane (TMS) as the internal standard.

${ }^{1} \mathrm{H}-\mathrm{NMR}\left(\mathrm{CD}_{3} \mathrm{OD}\right)$ 8: $7.6(\mathrm{H}-1 / 7, \mathrm{~d}, J=15.8 \mathrm{~Hz}) ; 7.24(\mathrm{H}-9 / 15, \mathrm{~s}) ; 7.14(\mathrm{H}-13 / 19, \mathrm{~d}, J=8.3 \mathrm{~Hz}) ; 6.85(\mathrm{H}-12 / 18$, $\mathrm{d}, J=8.2 \mathrm{~Hz}) ; 6.66(\mathrm{H}-2 / 6, \mathrm{~d}, J=15.8 \mathrm{~Hz}) ; 5.99(\mathrm{H}-4$ keto-enol form, $\mathrm{s}) ; 4.59(\mathrm{H}-4$ diketo form, $\mathrm{s}) ; 3.94(\mathrm{OMe}-10 / 6$, s). ${ }^{13} \mathrm{C}-\mathrm{NMR}\left(\mathrm{CD}_{3} \mathrm{OD}\right) \delta: 182.53$ (C-3); 182.58 (C-5); 148.24 (C-11/17); 147.18 (C-10/16); 139.88 (C-1/7); 126.35 (C-8/14); 121.86 (C-13/19); 120.02 (C-2/6); 114.33 (C-12/18); 109.51 (C-9/15); 99.73 (C-4); 54.22 (OMe-10/16).

The LC/MS analysis of TAE and curcumin as well as the mass and NMR spectra of curcumin are shown in the supplemental information.

Drugs. Bismuth (III) subsalicylate (batch number MKBV4704V) and type III mucin from porcine stomach were purchased from Sigma Aldrich (St Louis, MO, USA). Ranitidine was donated by HELM de México S. A. (batch number RH4570316, 99.6\% purity).

Preparation of the suspensions for curcumin and extract administration. Individual suspensions of the pure compound and the extract were prepared as follows to facilitate oral administration. One hundred milligrams of curcumin or TAE were combined with $0.25 \mathrm{~mL}$ of tween $80,0.25 \mathrm{~mL}$ of Span 20 and $1 \mathrm{~mL}$ of distilled water, and then stirred for $30 \mathrm{~min}$ at $338.2 \times \boldsymbol{g}$ with a homogenizer (ULTRATURAX ${ }^{\circledR}$ ). Afterwards, distilled water was added at $0.5 \mathrm{~mL} / \mathrm{min}$ to obtain a final volume of $10 \mathrm{~mL}$, and constant stirring was maintained for additional $90 \mathrm{~min}$. The suspensions showed viscoelastic characteristics and a non-Newtonian behavior. The particle size was measured ( 570 to $740 \mathrm{~nm}$ ) by a Zetasizer ZEN ZS 3600 (Malvern Panalytical Co., UK). The suspensions were stored at room temperature away from sunlight.

Rheological mucoadhesion evaluation. Mucoadhesion is the attachment of synthetic or biological macromolecules to a mucus layer by non-covalent molecular interactions ${ }^{29}$. The mucoadhesion of curcumin and the extract was evaluated using the rheological method previously reported by Hägerström et al. ${ }^{30}$. This methodology is based on the difference between the elastic $\left(G^{\prime}\right)$ and viscous $\left(\mathrm{G}^{\prime \prime}\right)$ moduli of the suspensions under oscillatory flow in a linear viscoelastic regime at $37^{\circ} \mathrm{C}$. The synergism parameters were estimated from Eqs. 1 and $2\left(\Delta \mathrm{G}^{\prime}\right.$ y $\left.\Delta \mathrm{G}^{\prime \prime}\right)$; where $\mathrm{G}^{\prime}$ sus and $\mathrm{G}^{\prime \prime}$ sus; $\mathrm{G}^{\prime} m u c$ and $\mathrm{G}^{\prime \prime} m u c$; and $\mathrm{G}^{\prime}$ mix $\left(\mathrm{G}^{\prime \prime} m i x\right)$ respectively represent the elastic and viscous moduli of the suspensions, mucin, and the mixture of mucin with curcumin or the extract. According to Hägerström et al. (2000), positive values of $\Delta \mathrm{G}^{\prime}$ and $\Delta \mathrm{G}^{\prime \prime}$ indicate there is an interaction between the components in the sample, thus mucoadhesion occurs ${ }^{30}$.

$$
\begin{gathered}
\Delta G^{\prime}=G_{m i x}^{\prime}-\left(G_{s u s}^{\prime}+G_{m u c}^{\prime}\right) \\
\Delta G^{\prime \prime}=G_{m i x}^{\prime \prime}-\left(G_{s u s}^{\prime \prime}+G_{m u c}^{\prime \prime}\right)
\end{gathered}
$$

The mucin was dispersed under magnetic stirring for $14 \mathrm{~h}$ at room temperature in a simulated gastric medium ( $\mathrm{HCl}$ aqueous solution, $\mathrm{pH}$ 1.6). The dispersion was then mixed with either curcumin or the extract suspension for a final mucin concentration of $5 \%(\mathrm{w} / \mathrm{w})$. The suspensions were diluted at $3 \%(\mathrm{w} / \mathrm{w})$ in the sample mixtures for the rheological evaluation. The mixtures were maintained under stirring for $30 \mathrm{~min}$ before testing. The rheological measurements were carried out using a controlled stress TA Instruments Discovery HR $3{ }^{\circledR}$ rheometer with concentric cylinder geometry $(21.96 \mathrm{~mm}$ external diameter, $20.38 \mathrm{~mm}$ inner diameter, $59.90 \mathrm{~mm}$ height and 500 $\mu \mathrm{m}$ of space between cylinders). The individual components and mixtures were characterized in the oscillatory flow mode from 1 to $100\left(\mathrm{rad} \mathrm{s}^{-1}\right)$ in the viscoelastic linear region ( $3 \%$ strain) at $37 \pm 1{ }^{\circ} \mathrm{C}$ (circulating water bath, Cole-Parmer Polystat, and Peltier AR-G2).

Animals. Male Wistar rats (250-300 g; 8 weeks old, $\mathrm{n}=258$ ) purchased from Envigo (Envigo RMS, S.A. de C.V., Mexico) were maintained at constant temperature $\left(22 \pm 2{ }^{\circ} \mathrm{C}\right)$ with free access to water and food. The rats were isolated and fasted in a metal cage with wire-net floor to avoid ingestion of feces and sawdust for 14 hours before the experiments; they had free access to water. 
Compliance with ethical standards. All applicable international, national and/or institutional guidelines for the care and use of animals were followed. Animal care and procedures were conducted in conformity with the Mexican Official Norm for Animal Care and Handing (NOM-062-ZOO-1999). This study was conducted under the supervision of the local Ethics Committee for the Use of Animals in Pharmacological and Toxicological Testing (CICUAL/147/16, 2016).

Ethanol-induced gastric ulcer in rats. The ethanol-induced ulcer model has been previously reported $^{31}$. The individual treatments (ranitidine, bismuth subsalicylate, curcumin or TAE) or combined (curcumin-ranitidine, curcumin-bismuth subsalicylate, TAE-ranitidine, TAE-bismuth subsalicylate) were orally administered $30 \mathrm{~min}$ before the alcohol-induced gastric damage (absolute ethanol, $1 \mathrm{~mL}$ ). It is important to notice that for the combined treatments, each compound was individually administered. First, the TAE or curcumin were given to the rats $5 \mathrm{~min}$ before the antiulcer drug. Then, the sequence was inverted, and the antiulcer drugs were administered $15 \mathrm{~min}$ before the TAE or curcumin. The control group was treated with the vehicle only. Two hours after ethanol administration, the rats were sacrificed in a $\mathrm{CO}_{2}$ chamber, and their stomachs dissected and fixed with $10 \mathrm{~mL}$ of paraformaldehyde $4 \%$ for $5 \mathrm{~min}$. Then, the stomachs were opened along the greater curvature and washed with a saline isotonic solution. A picture was taken using a digital microscope (Celestron 44302-A), to determine the damaged area $\left(\mathrm{mm}^{2}\right)$, using the software Image J (Rasband, W.S., Image J, U.S., National Institutes of Health, Bethesda, Maryland, USA, http://imagej.nih.gov/ij/, 1997-2004).

Gastroprotective effect and median-effect dose $(\mathrm{Dm})$ determination for single treatments. The orally-administered individual drugs were tested at different doses to build a dose-dependent effect curve. Curcumin and TAE were evaluated at $10^{-3}, 10^{-2}, 10^{-1}, 1$ and $10 \mathrm{mg} / \mathrm{kg}$; ranitidine at 1, 3, 10,30, 100 and $300 \mathrm{mg} / \mathrm{kg}$; and bismuth subsalicylate at $1,10,30,100$ and $177 \mathrm{mg} / \mathrm{kg}$.

The first step for the interaction analysis was to calculate the median-effect dose $(\mathrm{Dm})$ according to the Median-Effect Equation (Eq. 3) described by Chou and Talalay in 1976, for single drugs ${ }^{20}$. The Dm is defined as the dose when the affected fraction of the system $(f a)$ is 0.5 (the dose that shows $50 \%$ of gastroprotection against the ethanol-induced gastric damage). In Eq. 3, $D$ corresponds to each drug dose, $f u$ is the unaffected fraction ( $1-f a$ ), and $m$ denotes the graph shape ( $m=1$ is for hyperbolic, $m>1$ for sigmoidal and $m<1$ for flat-sigmoidal dose-effect curves).

$$
\frac{f a}{f u}=\left(\frac{D}{D m}\right)^{m}
$$

When plotting $x=\log (D) v s y=\log [f a /(1-f a)]$ (Eq. 4), it linearizes the dose-effect curves to easily calculate the $D m$ and the $m$ is calculated as the linear coefficient of the graph cited.

$$
\log \left[\frac{f a}{1-f a}\right]=m \log (D)-m \log (D m)
$$

Once the $D m$ was calculated for each drug, the combined treatments were evaluated according to the experimental design shown in Table 2. The combinations tested were TAE-ranitidine, TAE-bismuth subsalicylate, curcumin-ranitidine, and curcumin-bismuth subsalicylate at 1:1 considering the calculated $D m$ for each drug ${ }^{20}$.

Isobolographic analysis. The isobolographic analysis is a graphic representation of the doses of different drugs that have a specific effect, e.g., the median effect $(D m)$. A normalized isobologram is constructed from the $(D)_{1} /(D m)_{1}$ of drug $1 v s(D)_{2} /(D m)_{2}$ of drug 2 . Equation 5 presents the definition of additivity.

$$
\frac{(D)_{1}}{(D m)_{1}}+\frac{(D)_{2}}{(D m)_{2}}=1
$$

According to this equation, the maximum value for each drug is 1 in the $x$-and $y$-axis; a line called isobole or "additive line" connects these points and represents all the proportions in the combination that shows a theoretical additive effect. If the combination indicates there is an additive effect, the data points fall on this line. If the combination data points fall on the lower left or the upper right section, there is a superadditive (synergism) or subadditive (antagonism) interaction, respectively. The experimental points represent the median doses $(\mathrm{Dm})$ of the drug combination.

Equations 6 and 7 correspond to concave and convex isobole, respectively. The area between the concave and convex lines defines the isobologram additive area. In Eqs 6 and 7 " $a$ " values are the coordinates in the abscise (between 0 and 1 from the $(D)_{1} /(D m)_{1}$ ratio), and " $b$ " values are the coordinates in ordinate axis (between 0 and 1 from the $(D)_{2} /(D m)_{2}$ ratio).

$$
b=D m_{2}\left(\frac{D_{1}-a}{D m_{1}}\right)^{m_{1} / m_{2}}
$$

and

$$
b=D_{2}-\frac{D m_{2}}{\left(\frac{D m_{1}}{a}\right)^{m_{1} / m_{2}}}
$$


Combination-index determination. The combination index $(C I)$ for two drugs was determined according to Eq. $8^{20}$ :

$$
C I=\frac{(D)_{1}}{(D x)_{1}}+\frac{(D)_{2}}{(D x)_{2}}=\frac{(D)_{1}}{(D m)_{1}\left[\frac{f a}{1-f a}\right]^{1 / m}}+\frac{(D)_{2}}{(D m)_{2}\left[\frac{f a}{1-f a}\right]^{1 / m}}
$$

A $C I=1$ indicates there is an additive interaction; the superadditive and subadditive interactions correspond to $C I<1$ and $C I>1$. In Eq. 8, Dx represents the individual drug dose for reaching a specific percentage of effectiveness, and $D$ is the dose of each drug in the combination that produces this effectiveness.

One of the advantages of the CI-plot, compared to the isobologram, is that it allows to analyze all $\mathrm{fa}$ levels simultaneously with any number of drugs in the combination.

Statistical analysis. Statistically significant differences $(p<0.05)$ were interpreted as a synergism interaction if $(D)_{1} /(D m)_{1}+(D)_{2} /(D m)_{2}$ was significative $<1$ and as subadditive interaction if $(D)_{1} /(D m)_{1}+(D)_{2} /(D m)_{2}$ was significative $>1$. No statistical difference corresponded to an additive effect ${ }^{23}$.

The theoretical and experimental $D m$ of the combinations were compared based on the Mann-Whitney test to establish statistical difference $(p<0.05)$. The statistical difference between the treatments and the damaged control was determined by a One-way ANOVA and a post hoc Dunnett's test.

Received: 16 August 2019; Accepted: 25 October 2019;

Published online: 12 November 2019

\section{References}

1. Fasinu, P. S., Bouic, P. J. \& Rosenkranz, B. An overview of the evidence and mechanisms of herb-drug interactions. Front Pharmacol 3, 1-19 (2012).

2. Chavez, M. L., Jordan, M. A. \& Chavez, P. I. Evidence-based drug-herbal interactions. Life Sci 78, 2146-2157 (2006).

3. Choi, Y. H., Chin, Y. W. \& Kim, Y. G. Herb-drug interactions: Focus on metabolic enzymes and transporters. Arch Pharm Res 34, 1843-1863 (2011).

4. Dudhatra, G. B. et al. A Comprehensive Review on Pharmacotherapeutics of Herbal Bioenhancers. Sci. World J 2012, 637953 (2012).

5. Ravindran, P. N. Turmeric The genus Curcuma (ed. Ravindran, P. N.) (CRC Press Taylor and Francis Group, 2007).

6. Kim, D. C. et al. Curcuma longa Extract Protects against Gastric Ulcers by Blocking H2 Histamine Receptors. Biol Pharm Bull 28, 2220-2224 (2005).

7. Rahim, N. A. et al. Gastroprotective Effect of Ethanolic Extract of Curcuma xanthorrhiza Leaf against Ethanol-Induced Gastric Mucosal Lesions in Sprague-Dawley Rats. Biomed Res Int 2014, 416409 (2014).

8. Rouhollahi, E. et al. Evaluation of acute toxicity and gastroprotective activity of curcuma purpurascens BI. rhizome against ethanolinduced gastric mucosal injury in rats. BMC Complement Altern Med 14, 378 (2014).

9. Jurenka, J. Anti-inflammatory Properties of Curcumin, a Major Constituent of Curcuma longa: A Review of Preclinical and Clinical Research. Altern Med Rev 14, 141-153 (2009).

10. Epstein., J., Sanderson, I. R. \& MacDonald, T. T. Curcumin as a therapeutic agent: the evidence from in vitro, animal and human studies. Br J Nutr 103, 1545-1557 (2010).

11. Sharma, A. V., Ganguly, K., Paul, S., Maulik, N. \& Swarnakar, S. Curcumin Heals Indomethacin-Induced Gastric Ulceration by Stimulation of Angiogenesis and Restitution of Collagen Fibers via VEGF and MMP-2 Mediated Signaling. Antioxid Redox Signal 16, 351-362 (2012).

12. Shang, Y. J. et al. Antioxidant capacity of curcumin-directed analogues: Structure-activity relationship and influence of microenvironment. Food Chem 119, 1435-1442 (2010).

13. Barzegar, A. The role of electron-transfer and $\mathrm{H}$-atom donation on the superb antioxidant activity and free radical reaction of curcumin. Food Chem 135, 1369-1376 (2012).

14. Vimala, G. \& Gricilda Shoba, F. A Review on Antiulcer Activity of Few Indian Medicinal Plants. Int J Microbiol 2014, 519590 (2014).

15. Secretaría de Salud. Boletín Epidemiológico Semana 52. Sistema Nacional de Vigilancia Epidemiológica. Vol.35. Ciudad de México, México (2018).

16. Erickson, R. A., Bezabah, S., Jonas, G., Lifrak, E. \& Tarnawski, A. S. Chronic omeprazole treatment increases duodenal susceptibility to ethanol injury in rats. Digestive Diseases and Sciences 36, 897-904 (1991).

17. Tuskey, A. \& Peura, D. The use of $\mathrm{H} 2$ antagonists in treating and preventing NSAID-induced mucosal damage. Arthritis Res Ther 15, S6 (2013).

18. Tanaka, S., Guth, P. H., Carryl, O. R. \& Kaunitz, J. D. Cytoprotective effect of bismuth subsalicylate in indomethacin-treated rats is associated with enhanced mucus bismuth concentration. Aliment Pharmacol Ther 11, 605-612 (1997).

19. Bagchi, D. et al. Stress, diet and alcohol-induced oxidative gastrointestinal mucosal injury in rats and protection by bismuth subsalicylate. J Appl Toxicol 18, 3-13 (1998).

20. Chou, T.-C. Theoretical Basis, Experimental Design, and Computerized Simulation of Synergism and Antagonism in Drug Combination Studies. Pharmacol Rev 58, 621-681 (2006).

21. Ugalde, M. et al. Isobolographic analysis of the sedative interaction between six central nervous system depressant drugs and Valeriana edulis hydroalcoholic extract in mice. J Pharm Pharmacol 57, 631-639 (2005).

22. Balderas, J. L. et al. Pharmacodynamic interaction of the sedative effects of Ternstroemia pringlei (Rose) Standl. with six central nervous system depressant drugs in mice. J Ethnopharmacol 119, 47-52 (2008).

23. Rodríguez-Ramos, F., Andrade-Moreno, M. Á., Alfaro-Romero, A., Balderas-López, J. L. \& Navarrete, A. Gnaphaliin A and gnaphaliin B synergize the relaxant effect of salbutamol but not of ipratropium in Guinea pig trachea. J Pharm Pharmacol 68, 533-541 (2016).

24. Robert, A., Nezamis, J. E., Lancaster, C. \& Hanchar, A. J. Cytoprotection by prostaglandins in rats. Prevention of gastric necrosis produced by alcohol, $\mathrm{HCl}, \mathrm{NaOH}$, hypertonic $\mathrm{NaCl}$, and thermal injury. Gastroenterology 77, 433-443 (1979).

25. García-Guzmán, P. et al. Characterization of hybrid microparticles/Montmorillonite composite with raspberry-like morphology for Atorvastatin controlled release. Colloids Surfaces B Biointerfaces 167, 397-406 (2018).

26. Kwiecień, S., Brzozowski, T. \& Konturek, S. J. Effects of reactive oxygen species action on gastric mucosa in various models of mucosal injury. J Physiol Pharmacol 53, 39-50 (2002).

27. Péret-Almeida, L., Cherubino, A., Alves, R. J., Dufossé, L. \& Glória, M. B. A. Separation and determination of the physico-chemical characteristics of curcumin, demethoxycurcumin and bisdemethoxycurcumin. Food Res Int 38, 1039-1044 (2005). 
28. Cao, Y., Xu, R. X. \& Liu, Z. A high-throughput quantification method of curcuminoids and curcumin metabolites in human plasma via high-performance liquid chromatography/tandem mass spectrometry. J Chromatogr B Analyt Technol Biomed Life Sci 949-950, 70-78 (2014).

29. Madsen, F., Eberth, K. \& Smart, J. D. A rheological examination of the mucoadhesive/mucus interaction: the effect of mucoadhesive type and concentration. J Control Release 50, 167-78 (1998).

30. Hägerström, H., Paulsson, M. \& Edsman, K. Evaluation of mucoadhesion for two polyelectrolyte gels in simulated physiological conditions using a rheological method. EurJPharm Sci 9, 301-309 (2000).

31. Velázquez-Moyado, J. A. et al. Gastroprotective effect of diligustilide isolated from roots of Ligusticum porteri coulter \& rose (Apiaceae) on ethanol-induced lesions in rats. J Ethnopharmacol 174, 403-409 (2015).

\section{Acknowledgements}

Alejandra Orona-Ortiz acknowledges her fellowship from CONACyT México (257718). This investigation was supported by grants PAIP 5000-9143, DGAPA-UNAM IN213418 and CONACyT A1-S-9698. The authors thank Laboratorios MIXIM, S. A. de C.V. for the donation of Curcuma longa, which was cultivated in Tapachula, Chiapas, México.

\section{Author contributions}

A.O.-O. designed the research, wrote the manuscript and conducted the experiments of ethanol-induced damaged and mucoadhesion. L.M.-.T conducted and supervised the mucoadhesion experiments. J.V.-M. and E.P.-.P revised the manuscript and helped with the experimental design. J.C.T.C. designed the preparation of curcumin and T.A.E. suspensions. J.L.B.-L. helped with the isolation and identification of curcumin. M.J.B.-B. contributed with reagents. A.N.C. designed and supervised the research.

\section{Competing interests}

The authors declare no competing interests.

\section{Additional information}

Supplementary information is available for this paper at https://doi.org/10.1038/s41598-019-53089-2.

Correspondence and requests for materials should be addressed to A.N.

Reprints and permissions information is available at www.nature.com/reprints.

Publisher's note Springer Nature remains neutral with regard to jurisdictional claims in published maps and institutional affiliations.

(c) Open Access This article is licensed under a Creative Commons Attribution 4.0 International License, which permits use, sharing, adaptation, distribution and reproduction in any medium or format, as long as you give appropriate credit to the original author(s) and the source, provide a link to the Creative Commons license, and indicate if changes were made. The images or other third party material in this article are included in the article's Creative Commons license, unless indicated otherwise in a credit line to the material. If material is not included in the article's Creative Commons license and your intended use is not permitted by statutory regulation or exceeds the permitted use, you will need to obtain permission directly from the copyright holder. To view a copy of this license, visit http://creativecommons.org/licenses/by/4.0/.

(C) The Author(s) 2019 